\title{
3-乙/芳酰乙酰基吲哚的水相合成
}

\author{
胡小宇 海丰* 王文举* 姜思傲 刘 奇 何 洁
(白城师范学院化学学院 吉林白城 137000)
}

摘要 探讨了 4-十二烷基苯磺酸(DBSA)催化的 $\beta$-乙硫基- $\beta$-吲哚基- $\alpha, \beta$-不饱和酮(1)的水相水解反应，实现了 3 -乙/芳酰 乙酰基吲哚(2)的水相合成. 研究表明, 在回流和 $10 \mathrm{~mol} \% \mathrm{DBSA}$ 存在下, 1 有效地发生水相水解反应, 高产率合成产物 2 .

关键词 水相反应; 水解反应; 3-乙酰乙酰基吲哚; $\beta$-乙硫基- $\beta$-吲哚基- $\alpha, \beta$-不饱和酮

\section{Synthesis of 3-Ethanoyl/Aroylacetylindoles in Water}

\author{
Hu, Xiaoyu Yu, Haifeng* Wang, Wenju* Jiang, Siao Liu, Qi He, Jie \\ (College of Chemistry, Baicheng Normal University, Baicheng, Jilin 137000)
}

\begin{abstract}
Dodecylbenzenesulfonic acid (DBSA) catalyzed hydrolysis reaction of $\beta$-ethylthio- $\beta$-indolyl- $\alpha, \beta$-unsaturated ketones (1) in water to yield 3-ethanoyl/aroylacetylindoles (2) was studied. It showed that the hydrolysis of $\mathbf{1}$ in water smoothly occurred in the presence of $10 \mathrm{~mol} \%$ DBSA in reflux water, and $\mathbf{2}$ was obtained in excellent yield.
\end{abstract}

Keywords aqueous reaction; hydrolysis reaction; 3 -acetoacetylindole; $\beta$-ethylthio- $\beta$-indolyl- $\alpha, \beta$-unsaturated ketone

3-乙/芳酰乙酰基吲哚是一类重要的吲哚衍生物, 它不仅是许多具有良好生物活性吲哚衍生物的重要核 心结构 ${ }^{[13]}$, 而且也是进一步合成有重要生物活性吲哚 衍生物的重要中间体 ${ }^{[4,5]}$, 因此, 它的合成方法研究引起 人们的极大兴趣. 近二十年来, 发展了多种合成 3-乙/芳 酰乙酰基吲哚的方法, 主要包括吲哚的直接 Friedel-

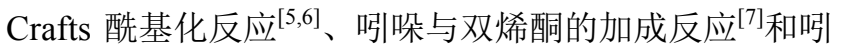
哚与酯的碱性条件下的缩合反应 ${ }^{[8,9]}$. 最近, 我们研究组 基于易制备的 $\beta$-乙硫基 $-\beta$-吲哚基- $\alpha$, $\beta$-不饱和羰基化合 物的酸性水解反应, 有效合成了 3 -吲哚基-3-氧代丙酸 酯 ${ }^{[10,11]}$ 和 3-乙/芳酰乙酰基吲哚 ${ }^{[12]}$. 虽然这些方法都能 有效合成 3-乙/芳酰乙酰基吲哚衍生物, 但从绿色化学 观点考虑, 它们都是在乙腈、四氢呋喃和二氯甲烷等有 机溶剂中进行的反应, 大量有机溶剂的使用和排放能够 导致环境污染和安全问题, 与绿色化学理念相悖. 因此, 基于绿色化学理念, 发展绿色的 3-乙/芳酰乙酰基吲哚 衍生物的水相合成是非常必要的. 文献调研可知, 现在 还没有关于 3-乙/芳酰乙酰基吲哚的水相合成研究.
水相有机反应是绿色化学的重要研究内容之一, 因 为水相有机反应具有操作简单、显著地减少有毒有害溶 剂的使用和排放及环境友好等优点, 所以引起了许多有 机合成化学家的广泛关注 ${ }^{[13 \sim 19]}$. 近些年来, 我们研究组 一直进行基于二硫缩烯酮及衍生物的水相反应研究, 已 经成功实现了无气味的 $\alpha$-羰基二硫缩烯酮作代硫醇试 剂与醛或酮的水相硫缩醛酮化反应 ${ }^{[20]}$ 、二硫缩烯酮与醇 的水相 Friedel-Crafts 烷基化反应 ${ }^{[21]}$ 和二硫缩烯酮的水 相水解反应 ${ }^{[22]}$. 作为系列工作, 在实现了硫酸催化 $\beta$-乙 硫基- $\beta$-吲哚基- $\alpha, \beta$-不饱和酮有机相水解, 有效合成 3乙/芳酰乙酰基吲哚的基础上 ${ }^{[12]}$, 我们最近系统研究了 $\beta$-乙硫基- $\beta$-吲哚基- $\alpha, \beta$-不饱和酮的水相水解反应，成功 实现绿色的 3-乙/芳酰乙酰基吲哚的水相合成.

\section{1 结果与讨论}

$\beta$-乙硫基- $\beta$-吲哚基 $-\alpha, \beta$-不饱和酮(1)是具有多反应 活性中心的合成有潜在生物活性吲哚衍生物的重要中 间体，它可以通过在酸性条件下由重要的合成中间体 $\alpha$ -

\footnotetext{
* Corresponding authors. E-mail: yuhf68105@sina.com; wangwj3309@126.com Received April 3, 2019; revised June 18, 2019; published online July 9, 2019. Project supported by the Foundation of Science and Technology Research Projects of the 13th Five-Year Plan of Jilin Provincial Department of Education (No. 2016037).

吉林省教育厅十三五计划科技(No. 2016037)资助项目.
} 
羰基二硫缩烯酮 ${ }^{[23 ~ 26]}$ 与吲哚的选择性脱硫偶联反应制 备 ${ }^{[27,28]}$.

\section{1 反应条件优化}

在研究中, 以 $\mathbf{1} \mathbf{a}$ 的水解反应为例, 进行反应条件优 化. 根据以前的工作 ${ }^{[20 ~ 22]}$, 选择 4-十二烷基苯磺酸 (DBSA)作为催化剂, 实验结果概括在表 1 中. 研究表 明, DBSA 的用量和反应温度对 1a 的水解反应有重要的 影响. 在回流条件下, 水中没有 DBSA 时, 1a 不发生水 解反应(表 1, Entry 1); 当 DBSA 的量等于或大于 10 $\mathrm{mol} \%$ 时, 水解反应有效发生, 高产率制得 3-乙酰乙酰 基吲槑(2a) (表 1, Entries 2 4), 而 DBSA 的量降低到 $7.5 \mathrm{~mol} \%$ 时，反应效率明显降低(表 1 , Entries 5,6). 当反 应温度降低到 $90{ }^{\circ} \mathrm{C}$ 时, 即使延长反应时间, 产物 $2 \mathbf{a}$ 的 产率明显降低(表 1, Entries 7,8). 根据上述结果, $\beta$-乙硫 基- $\beta$-吲哚 基- $\alpha, \beta$-不饱和酮水相水解的最佳反应条件 为: $10 \mathrm{~mol} \% \mathrm{DBSA}$ 为催化剂, 回流.

\section{2 绿色的 3-乙/芳酰乙酰基吲哚的水相合成}

如表 2 所示, 在最佳反应条件下, $\beta$-乙硫基- $\beta$-吲哚
表 1 反应条件优化 ${ }^{a}$

Table 1 Screening of reaction conditions

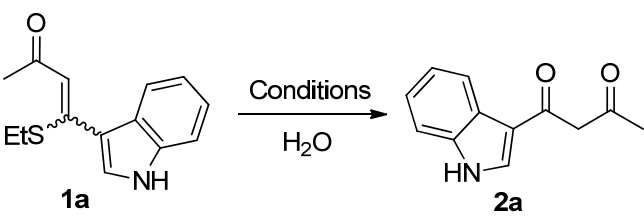

\begin{tabular}{ccccc}
\hline Entry & DBSA $/ \mathrm{mol} \%$ & $T /{ }^{\circ} \mathrm{C}$ & Time $/ \mathrm{h}$ & Yield $^{b} / \%$ \\
\hline 1 & 0 & Reflux & 20 & 0 \\
2 & 20 & Reflux & 6 & 95 \\
3 & 15 & Reflux & 8 & 94 \\
4 & 10 & Reflux & 9 & 95 \\
5 & 7.5 & Reflux & 15 & 90 \\
6 & 7.5 & Reflux & 20 & 92 \\
7 & 10 & 90 & 9 & 88 \\
8 & 10 & 90 & 17 & 92 \\
\hline
\end{tabular}

${ }^{a}$ Reaction conditions: 1a $(0.5 \mathrm{mmol}), \mathrm{H}_{2} \mathrm{O}$ solvent $(1 \mathrm{~mL}){ }^{b}$ Isolated yield.

基- $\alpha, \beta$-不饱和酮(1)的水相水解反应能有效进行，高产 率制得 1-吲哚基-1,3-丁二酮 $\mathbf{2 a} \sim \mathbf{2} \mathbf{j}$ 和 3-苯基-1-吲哚基1,3-丙二酮 $\mathbf{2 k} \sim \mathbf{2} \mathbf{p}$. 反应物 $\mathbf{1}$ 中吲哚环的 5, 6, 7 位和芳 环上的供电子或吸电子取代基对水解反应没有明显的

表 22 的水相合成 ${ }^{a}$

Table 2 Synthesis of 2 in water<smiles>[R]C(=O)C=C([Se])c1c([R])n([R])c2cc([R])ccc12</smiles><smiles>[R4]C(=O)CC(=O)c1c([R])n([R])c2cc[R4]([H])cc12</smiles><smiles></smiles>

2a, $95 \%$, Enol:Keto $=10: 9$<smiles>COc1ccc2[nH]cc(C(=O)CC(C)=O)c2c1</smiles>

2e, $95 \%$, Enol:Keto $=1: 1$<smiles>CCn1cc(C(=O)CC(C)=O)c2ccccc21</smiles>

2i, $92 \%$, Enol:Keto $=4: 3$

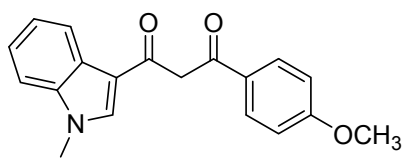

2m, $93 \%$, Enol:Keto $=2: 1$

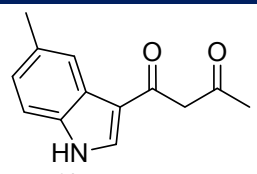

2b, $92 \%$, Enol:Keto $=5: 4$

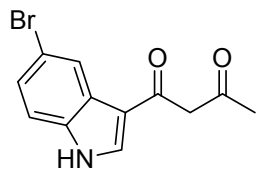

2f, $94 \%$, Enol:Keto $=1: 1$<smiles>CC(=O)CC(=O)c1cn(Cc2ccccc2)c2ccccc12</smiles>

2j, $96 \%$, Enol:Keto = 5:3

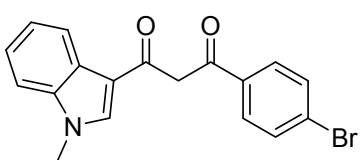

2n, 93\%, Enol:Keto = 6:1<smiles>CC(=O)CC(=O)c1c[nH]c2cc(C)ccc12</smiles>

2c, $94 \%$, Enol:Keto $=5: 4$<smiles>CC(=O)CC(=O)c1c(C)[nH]c2ccccc12</smiles>

$\mathbf{2 g}^{b}, 91 \%$, Enol:Keto $=3: 1$

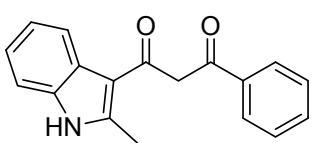

$\mathbf{2} \mathbf{k}^{b}, 90 \%$, Enol:Keto $=9: 1$

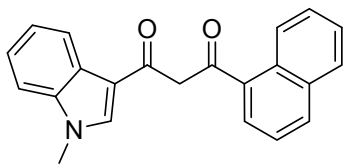

2o, $92 \%$, Enol:Keto = 9:1<smiles>CC(=O)CC(=O)c1c[nH]c2c(C)cccc12</smiles>

2d, $91 \%$, Enol:Keto $=5: 4$<smiles>CC(=O)CC(=O)c1cn(C)c2ccccc12</smiles>

2h, 94\%, Enol:Keto $=5: 4$

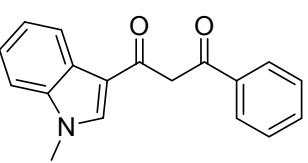

2I, $96 \%$, Enol:Keto $=4: 1$

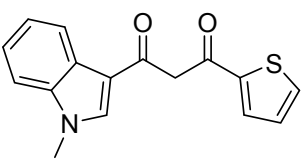

2p, 93\%, Enol:Keto $=5: 4$

${ }^{a}$ Isolated yield; enol/keto ratio of 2 determined by ${ }^{1} \mathrm{H}$ NMR; ${ }^{b} 30$ mol $\%$ of DBSA used, $24 \mathrm{~h}$. 
影响, 然而吲哚环的 2 位的取代基, 由于空间位阻的作 用, 对水解反应影响较大, 需要过量的 DBSA 和较长的 反应时间(例如, $\mathbf{2 g}$ 和 $\mathbf{2 k}$ 的制备). 从 ${ }^{1} \mathrm{H} N \mathrm{NMR}$ 和 IR 谱图 可知, 制得的 2 是烯醇式和酮式的混合物, 烯醇式异构 体是主要的. 2 的烯醇式可有 $2 \mathrm{~A}$ 和 $2 \mathrm{~B}$ 两种形式(图 1), 在研究中, 我们对产物 2 的稳定烯醇式结构进行探讨.<smiles>[R]C(=O)/C=C(/O)c1c([R])n([R])c2ccccc12</smiles><smiles>[R]C(O)=CC(=O)c1c([R])n([R])c2cc([R4])ccc12</smiles>

图 1 烯醇式结构 2

Figure 1 Proposed enol structures of 2

王群等 ${ }^{[29]}$ 采用密度泛函理论方法对苯甲酰丙酮异 构化为两种不同烯醇产物的反应机理进行了详细的探 究. 通过对键长的变化和能量的分析, 发现苯甲酰丙酮 的烯醇式的最稳定结构是离苯环远的羰基形成的烯醇 式. 在研究中, 我们分别以 $\mathbf{2 h}$ 和 21 的烯醇式构筑模型 (图 2), 采用密度泛函理论方法计算, 在 M06-2x/6$311 \mathrm{G}^{*}$ 水平下计算了它们的单点能和体系自由能, 从而 确定 2 的烯醇式的稳定结构, 计算结果整理于表 3 中. 从表 3 可知, $2 \mathrm{hB}$ 的单点能量略小于 $2 \mathrm{hA}, 2 \mathrm{lB}$ 的单点能 量小于 2lA, 因此两类具有烯醇式结构的分子中, $2 \mathrm{hB}$ 和 2IB 更稳定. 为了更精确地判断同分异构体结构的稳定 性, 我们又进行了它们的自由能的计算, 结果显示与单 点能结论相一致, $2 \mathrm{hB}$ 和 $2 \mathrm{IB}$ 的自由能分别比相应的小, 更稳定. 因此, 根据密度泛函理论方法计算的结果, 产 物 2 的烯醇式最稳定结构是 2 B.

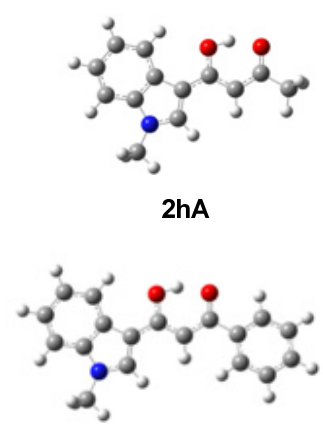

2IA

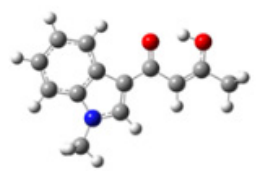

$2 \mathrm{hB}$

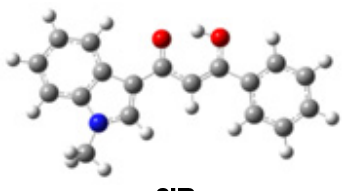

2IB
图 2 在 B3LYP-D3/6-31G*水平下优化得到的 $\mathbf{2 h A} \sim 2 \mathrm{IB}$ 分子 的几何结构

Figure 2 Optimized geometric structures of the $\mathbf{2 h A} \sim 2$ IB based on B3LYP-D3/6-31G * level

\section{3 扩展性实验和水循环实验}

以 $2 \mathbf{a}$ 的合成为例, 进行该合成方法的扩展性实验 (Eq. 1)和水循环实验(表 4). 研究表明, 在最佳反应条件 在水循环实验中, 发现水循环使用两次时, 反应效率变
表 3 在 $\mathrm{M} 06-2 \mathrm{x} / 6-311 \mathrm{G}$ *水平下计算的 $\mathbf{2 h A} \sim 2 \mathrm{2IB}$ 分子的能量 Table 3 Calculated energies of $\mathbf{2 h A} \sim 2$ IB based on M06-2x/6$311 \mathrm{G}^{*}$ level

\begin{tabular}{ccc}
\hline Item & Single-point energy/eV & Free energy/Hartree \\
\hline 2hA & -19273.19 & -708.09621 \\
2hB & -19273.28 & -708.09695 \\
2lA & -24489.64 & -899.74782 \\
2IB & -24489.69 & -899.75220 \\
\hline
\end{tabular}

下, $5 \mathrm{mmol}(1.225 \mathrm{~g})$ 的 $1 \mathrm{a}$ 有效发生水解反应，制得 4.6 mmol (0.93 g) 2a, 产率达到 92\%.

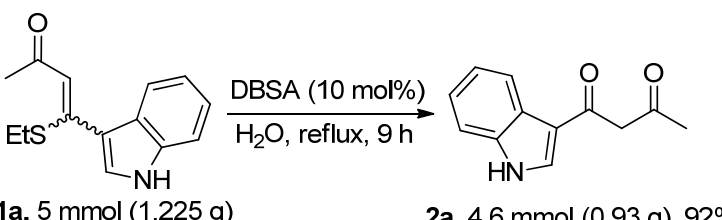

表 4 水循环实验

Table 4 Cycle experiment of water

\begin{tabular}{ccc}
\hline Run & $t /$ h & Yield $/ \%$ \\
\hline 1 & 10 & 94 \\
2 & 13 & 92 \\
3 & 20 & 83 \\
4 & 30 & 70 \\
\hline
\end{tabular}

化不大，产物 $\mathbf{2 a}$ 的产率仍然能达到 $92 \%$ 以上. 进一步实 验时，反应效率明显降低，不仅近反应时间延长，而且 $2 \mathbf{a}$ 的产率显著降低.

\section{2 结论}

首次实现了基于 DBSA 催化的 $\beta$-乙硫基- $\beta$-吲哚基$\alpha, \beta$-不饱和酮(1)水相水解反应的 3-乙/芳酰乙酰基吲哚 (2)的水相合成. 该方法不仅产物产率高，后处理简单， 而且显著减少了有毒有害的有机溶剂的使用和排放，契 合绿色化学理念，对环境保护有重要意义.

\section{3 实验部分}

\section{1 仪器与试剂}

XRC-1 显微熔点测定仪(温度计未经校正); Unity-600 或 400 核磁共振仪 $\left(\mathrm{CDCl}_{3}\right.$ 溶剂, TMS 为内标); AB-SCIEX Triple TOF 4600 高分辨质谱仪(ESI-MS/ MS); Tensor27 型傅立叶变换红外光谱仪; 所用试剂均 为分析纯. $\beta$-乙硫基- $\beta$-吲哚基- $\alpha, \beta$-不饱和酮(1)按文献 $[27,28]$ 报道的方法制备.

\section{2 实验方法}

以 $2 \mathrm{a}$ 的合成为例, 在 $20 \mathrm{~mL}$ 反应管中加入 $1(0.5$ $\mathrm{mmol}, 122.5 \mathrm{mg}$ )和 $1 \mathrm{~mL}$ 浓度为 $0.05 \mathrm{~mol} / \mathrm{L}$ 的 DBSA 水 溶液. 回流 $9 \mathrm{~h}$, 薄层色谱(TLC)检测底物消失后, 停止 
加热, 冷却到室温, 析出晶体后, 减压抽滤, 水洗和干 燥得到白色晶体 2a (95.5 mg), 产率 95\%. 用相同方法合 成 $\mathbf{2 b} \sim \mathbf{2 p}$.

1-[3-(1H-吲哚基)]-1,3-丁二酮(2a): 白色固体. m.p. $132 \sim 133{ }^{\circ} \mathrm{C} ;{ }^{1} \mathrm{H}$ NMR $\left(\mathrm{CDCl}_{3}, 600 \mathrm{MHz}\right) \delta$ : For enol-2a: 9.01 (br, 1H), 8.39 (t, $J=8.2 \mathrm{~Hz}, 1 \mathrm{H}), 7.85$ (s, 1H), $7.32 \sim$ $7.28(\mathrm{~m}, 3 \mathrm{H}), 6.02(\mathrm{~s}, 1 \mathrm{H}), 2.14(\mathrm{~s}, 3 \mathrm{H})$; For keto-2a: 9.19 (br, $1 \mathrm{H}), 8.27$ (t, $J=8.2 \mathrm{~Hz}, 1 \mathrm{H}), 7.82(\mathrm{~s}, 1 \mathrm{H}), 7.42 \sim 7.40$ $(\mathrm{m}, 3 \mathrm{H}), 3.98$ (s, 2H), $2.32(\mathrm{~s}, 3 \mathrm{H}) ;{ }^{13} \mathrm{C} \mathrm{NMR}\left(\mathrm{CDCl}_{3}, 150\right.$ MHz) $\delta$ : For enol-2a: 187.0, 184.8, 136.7, 133.3, 129.6, 124.2, 123.6, 122.4, 121.9, 111.9, 97.1, 24.4; For keto-2a: $203.7,188.1,136.5,125.5,125.2,123.8,123.2,122.8$, 122.4, 111.7, 57.1, 30.5; IR (KBr) v: 3261,3122, 2973, 2948, 2859. 1739, 1632, 1580, 1426, 1376, $1237 \mathrm{~cm}^{-1}$; HRMS cacld for $\mathrm{C}_{12} \mathrm{H}_{12} \mathrm{NO}_{2}[\mathrm{M}+\mathrm{H}]^{+}$202.0863, found 202.0868..

1-[3-(5-甲基- $1 H$-吲哚基)]-1,3-丁二酮(2b): 白色固 体. m.p. $223 \sim 224{ }^{\circ} \mathrm{C} ;{ }^{1} \mathrm{H} \mathrm{NMR}\left(\mathrm{CDCl}_{3}, 600 \mathrm{MHz}\right) \delta$ : For enol-2b: 8.78 (br, 1H), 8.19 (s, 1H), 7.79 (d, $J=2.2 \mathrm{~Hz}$, $1 \mathrm{H}), 7.29$ (t, $J=7.6 \mathrm{~Hz}, 2 \mathrm{H}), 6.00(\mathrm{~s}, 1 \mathrm{H}), 2.47$ (s, 3H), 2.14 (s, 3H); For keto-2b: 8.92 (br, 1H), 8.07 (s, 1H), 7.83 $(\mathrm{d}, J=2.2 \mathrm{~Hz}, 1 \mathrm{H}), 7.11(\mathrm{t}, J=8.1 \mathrm{~Hz}, 2 \mathrm{H}), 3.97(\mathrm{~s}, 2 \mathrm{H})$, 2.49 (s, 3H), 2.32 (s, 3H); ${ }^{13} \mathrm{C} \mathrm{NMR}\left(\mathrm{CDCl}_{3}, 150 \mathrm{MHz}\right) \delta$ : For enol-2b: 186.2, 185.2, 133.1, 129.5, 125.7, 125.1, 122.0, 122.4, 121.6, 111.3, 96.9, 24.1, 21.6; For keto-2b: 203.5, 187.9, 134.9, 134.7, 132.9, 125.6, 125.4, 125.3, 122.0, 111.1, 57.1, 30.3, 21.6; IR (KBr) v: 3420, 3148, $3046,2921,2846,1711,1624,1557,1435,1301,1240 \mathrm{~cm}$ ${ }^{-1}$; HRMS cacld for $\mathrm{C}_{13} \mathrm{H}_{14} \mathrm{NO}_{2}[\mathrm{M}+\mathrm{H}]^{+} 216.1019$, found 216.1010 .

1-[3-(6-甲基-1H-吲哚基)]-1,3-丁二酮(2c): 白色固 体. m.p. $133 \sim 134{ }^{\circ} \mathrm{C} ;{ }^{1} \mathrm{H}$ NMR $\left(\mathrm{CDCl}_{3}, 600 \mathrm{MHz}\right) \delta$ : For enol-2c: 8.77 (br, 1H), 8.12 (d, $J=8.1 \mathrm{~Hz}, 1 \mathrm{H}), 7.75$ (s, $1 \mathrm{H}), 7.19$ (s, 1H), 7.12 (d, $J=8.5 \mathrm{~Hz}, 1 \mathrm{H}), 6.00$ (s, 1H), $2.46(\mathrm{~s}, 3 \mathrm{H}), 2.13$ (s, 3H); For keto-2c: 8.93 (br, 1H), 8.22 $(\mathrm{d}, J=8.0 \mathrm{~Hz}, 1 \mathrm{H}), 7.77(\mathrm{~s}, 1 \mathrm{H}), 7.17(\mathrm{~s}, 1 \mathrm{H}), 7.14$ (d, $J=$ $8.5 \mathrm{~Hz}, 1 \mathrm{H}), 3.96(\mathrm{~s}, 2 \mathrm{H}), 2.45$ (s, 3H), $2.32(\mathrm{~s}, 3 \mathrm{H}) ;{ }^{13} \mathrm{C}$ NMR $\left(\mathrm{CDCl}_{3}, 150 \mathrm{MHz}\right) \delta$ : For enol-2c: $186.9,184.8$, $133.6,132.8,129.1,124.9,124.2,121.9,121.5,111.7$, 97.0, 24.4, 21.7; For keto-2c: 203.7, 188.0, 137.1, 137.0, 134.3, 123.2, 123.0, 117.8, 114.9, 111.6, 57.1, 30.5, 21.7; IR (KBr) v: 3395, 3118, 2964, 2923, 2857, 1707, 1594, $1530,1442,1339,1242 \mathrm{~cm}^{-1}$; HRMS cacld for $\mathrm{C}_{13} \mathrm{H}_{14} \mathrm{NO}_{2}$ $[\mathrm{M}+\mathrm{H}]^{+}$216.1019, found 216.1025.

1-[3-(7-甲基-1 $H$-吲哚基)]-1,3-丁二酮(2d): 白色固
体. m.p. $143 \sim 144{ }^{\circ} \mathrm{C}$; ${ }^{1} \mathrm{H}$ NMR $\left(\mathrm{CDCl}_{3}, 600 \mathrm{MHz}\right) \delta$ : For enol-2d: $8.86(\mathrm{br}, 1 \mathrm{H}), 8.09$ (d, $J=8.0 \mathrm{~Hz}, 1 \mathrm{H}), 7.81$ (s, $1 \mathrm{H}), 7.23 \sim 7.20(\mathrm{~m}, 2 \mathrm{H}), 6.03(\mathrm{~s}, 1 \mathrm{H}), 2.50$ (s, 3H), 2.14 (s, 3H); For keto-2d: 9.03 (br, $1 \mathrm{H}), 8.20(\mathrm{~d}, J=7.9 \mathrm{~Hz}$, 1H), $7.84(\mathrm{~s}, 1 \mathrm{H}), 7.10 \sim 7.08(\mathrm{~m}, 2 \mathrm{H}), 3.97$ (s, 2H), 2.49 $(\mathrm{s}, 3 \mathrm{H}), 2.32(\mathrm{~s}, 3 \mathrm{H}) ;{ }^{13} \mathrm{C} \mathrm{NMR}\left(\mathrm{CDCl}_{3}, 150 \mathrm{MHz}\right) \delta$ : For enol-2d: 187.2, 184.7, 136.3, 133.0, 129.7, 124.8, 124.2, 122.6, 119.5, 115.4, 97.1, 24.3, 16.6; For keto-2d: 203.7, $188.1,136.1,125.1,124.2,123.4,121.1,121.0,119.9$, 118.1, 57.2, 30.5, 16.6; IR (KBr) v: 3206, 3114, 3056, 2928, 2876, 1724, 1623, 1594, 1527, 1438, 1300, 1244 $\mathrm{cm}^{-1}$; HRMS Cacld for $\mathrm{C}_{13} \mathrm{H}_{14} \mathrm{NO}_{2}[\mathrm{M}+\mathrm{H}]^{+}$216.1019, found 216.1022 .

1-[3-(5-甲氧基- $1 H$-吲哚基)]-1,3-丁二酮(2e): 白色 固体. m.p. $123 \sim 124{ }^{\circ} \mathrm{C} ;{ }^{1} \mathrm{H}$ NMR $\left(\mathrm{CDCl}_{3}, 600 \mathrm{MHz}\right) \delta$ : For enol-2e: 8.93 (br, 1H), $7.80(\mathrm{~s}, 1 \mathrm{H}), 7.76$ (d, $J=2.7$ $\mathrm{Hz}, 1 \mathrm{H}), 7.27$ (t, $J=7.2 \mathrm{~Hz}, 1 \mathrm{H}), 6.92$ (d, $J=8.8 \mathrm{~Hz}, 1 \mathrm{H})$, $5.95(\mathrm{~s}, 1 \mathrm{H}), 3.86(\mathrm{~s}, 3 \mathrm{H}), 2.11(\mathrm{~s}, 3 \mathrm{H})$; For keto-2e: 9.10 (br, 1H), 7.87 (s, 1H), 7.80 (d, $J=2.2 \mathrm{~Hz}, 1 \mathrm{H}), 7.29$ (t, $J=$ $8.1 \mathrm{~Hz}, 1 \mathrm{H}), 6.92(\mathrm{~d}, J=8.8 \mathrm{~Hz}, 1 \mathrm{H}), 3.96$ (s, 2H), 3.89 (s, $3 \mathrm{H}), 2.33(\mathrm{~s}, 3 \mathrm{H}) ;{ }^{13} \mathrm{C} \mathrm{NMR}\left(\mathrm{CDCl}_{3}, 150 \mathrm{MHz}\right) \delta$ : For enol-2e: 186.1, 185.1, 156.1, 131.3, 129.8, 126.2, 115.0, 114.0, 112.5, 103.5, 96.8, 55.8, 23.8; For keto-2e: 203.7, $188.1,156.7$, 133.4, 131.5, 126.3, 117.6, 114.8, 112.5, 103.7, 56.9, 55.9, 30.5; IR (KBr) v: 3249, 3128, 2960, 2924, 2837, 1716, 1635, 1541, 1457, 1338, $1266 \mathrm{~cm}^{-1}$; HRMS cacld for $\mathrm{C}_{13} \mathrm{H}_{14} \mathrm{NO}_{3}[\mathrm{M}+\mathrm{H}]^{+}$232.0968, found 232.0960 .

1-[3-(5-溴-1H-吲哚基)]-1,3-丁二酮(2f): 白色固体. m.p. $166 \sim 167{ }^{\circ} \mathrm{C} ;{ }^{1} \mathrm{H}$ NMR $\left(\mathrm{CDCl}_{3}, 600 \mathrm{MHz}\right) \delta$ : For enol-2f: 8.72 (br, 1H), 8.45 (s, 1H), $7.82(\mathrm{~s}, 1 \mathrm{H}), 7.39$ (t, $J=8.3 \mathrm{~Hz}, 1 \mathrm{H}), 7.28$ (d, $J=8.8 \mathrm{~Hz}, 1 \mathrm{H}), 5.97$ (s, 1H), 2.15 (s, 3H); For keto-2f: 8.84 (br, 1H), 8.56 (s, 1H), 7.90 (s, $1 \mathrm{H}), 7.39$ (t, $J=8.4 \mathrm{~Hz}, 1 \mathrm{H}), 7.29$ (d, $J=8.8 \mathrm{~Hz}, 1 \mathrm{H}), 3.98$ $(\mathrm{s}, 2 \mathrm{H}), 2.33(\mathrm{~s}, 3 \mathrm{H}) ;{ }^{13} \mathrm{C} \mathrm{NMR}\left(\mathrm{CDCl}_{3}, 150 \mathrm{MHz}\right) \delta$ : For enol-2f: $186.7,184.5,135.1,129.8,126.9,126.6,124.7$, 117.3, 114.9, 113.0, 96.9, 24.2; For keto-2f: 203.1, 187.5, $134.9,127.3,127.0,125.1,116.8,115.9,112.9$, 112.7, 57.2, 30.4; IR (KBr) v: 3421, 3220, 2925, 2857, 1716, 1634, 1541, 1456, 1324, $1233 \mathrm{~cm}^{-1}$; HRMS cacld for $\mathrm{C}_{12} \mathrm{H}_{11} \mathrm{BrNO}_{2}[\mathrm{M}+\mathrm{H}]^{+}$279.9968, found 279.9970.

1-[3-(2-甲基-1 $H$-吲哚基)]-1,3-丁二酮(2g): 白色固 体, m.p. $163 \sim 164{ }^{\circ} \mathrm{C} ;{ }^{1} \mathrm{H}$ NMR $\left(\mathrm{CDCl}_{3}, 600 \mathrm{MHz}\right) \delta$ : For enol-2g: 8.56 (br, $1 \mathrm{H}), 7.96$ (d, $J=7.6 \mathrm{~Hz}, 1 \mathrm{H}), 7.24 \sim 7.19$ $(\mathrm{m}, 3 \mathrm{H}), 6.06(\mathrm{~s}, 1 \mathrm{H}), 2.71(\mathrm{~s}, 3 \mathrm{H}), 2.18(\mathrm{~s}, 3 \mathrm{H})$; For ke- 
to-2g: 8.78 (br, 1H), 7.78 (d, $J=7.8 \mathrm{~Hz}, 1 \mathrm{H}), 7.32 \sim 7.30$ (m, 3H), 4.11 (s, 2H), 2.71 (s, 3H), 2.35 (s, 3H); ${ }^{13} \mathrm{C}$ NMR $\left(\mathrm{CDCl}_{3}, 150 \mathrm{MHz}\right) \delta$ : For enol-2g: 190.6, 183.6, 141.8, 135.0, 126.4, 122.5, 121.8, 120.5, 111.1, 111.8, 98.7, 25.4, 15.1; For keto-2g: 204.1, 190.4, 145.0, 134.5, 126.4, 122.8, 122.5, 120.4, 114.1, $110.158 .1,30.8,15.6$; IR (KBr) $v$ : 3210, 3187, 2975, 2938, 2854, 1734, 1613, 1543, 1487, 1361, $1220 \mathrm{~cm}^{-1}$; HRMS cacld for $\mathrm{C}_{13} \mathrm{H}_{14} \mathrm{NO}_{2}[\mathrm{M}+\mathrm{H}]^{+}$ 216.1019, found 216.1017.

1-[3-(1-甲基吲哚基)]-1,3-丁二酮(2h): 白色固体. m.p. $105 \sim 106{ }^{\circ} \mathrm{C} ;{ }^{1} \mathrm{H}$ NMR $\left(\mathrm{CDCl}_{3}, 600 \mathrm{MHz}\right) \delta$ : For enol-2h: 8.37 (d, $J=7.8 \mathrm{~Hz}, 1 \mathrm{H}), 7.72(\mathrm{~s}, 1 \mathrm{H}), 7.36 \sim 7.29$ (m, 3H), $5.99(\mathrm{~s}, 1 \mathrm{H}), 3.84(\mathrm{~s}, 3 \mathrm{H}), 2.12(\mathrm{~s}, 3 \mathrm{H})$; For keto2h: 8.25 (d, $J=7.4 \mathrm{~Hz}, 1 \mathrm{H}), 7.78$ (s, 1H), 7.36 7.29 (m, $3 \mathrm{H}), 3.95$ (s, 2H), 3.85 (s, 3H), 2.32 (s, 3H); ${ }^{13} \mathrm{C}$ NMR $\left(\mathrm{CDCl}_{3}, 150 \mathrm{MHz}\right) \delta$ : For enol-2h: 186.5, 184.4, 137.8, 133.7, 126.1, 123.9, 123.2, 122.7, 122.3, 110.0, 96.9, 33.8, 24.3; For keto-2h: 203.5, 187.0, 137.6, 137.2, 126.4, 123.2, 122.1, 116.2, 113.4, 109.9, 57.7, 33.7, 30.2; IR (KBr) v: 3423, 3117, 3056, 2926, 2857, 1735, 1636, 1540, 1472, $1367,1234 \mathrm{~cm}^{-1}$; HRMS cacld for $\mathrm{C}_{13} \mathrm{H}_{14} \mathrm{NO}_{2}[\mathrm{M}+\mathrm{H}]^{+}$ 216.1019, found 216.1025 .

1-[3-(1-乙基吲哚基)]-1,3-丁二酮(2i): 白色固体. m.p. $156 \sim 157{ }^{\circ} \mathrm{C} ;{ }^{1} \mathrm{H}$ NMR $\left(\mathrm{CDCl}_{3}, 600 \mathrm{MHz}\right) \delta$ : For enol-2i: $8.26 \sim 8.24(\mathrm{~m}, 1 \mathrm{H}), 7.76(\mathrm{~s}, 1 \mathrm{H}), 7.30 \sim 7.27(\mathrm{~m}$, $3 \mathrm{H}), 5.99(\mathrm{~s}, 1 \mathrm{H}), 4.19 \sim 4.14(\mathrm{~m}, 2 \mathrm{H}), 2.11(\mathrm{~s}, 3 \mathrm{H}), 1.50$ (t, $J=7.5 \mathrm{~Hz}, 3 \mathrm{H})$; For keto-2i: $8.38 \sim 8.36(\mathrm{~m}, 1 \mathrm{H}), 7.81$ (s, 1H), $7.36 \sim 7.34(\mathrm{~m}, 3 \mathrm{H}), 4.19 \sim 4.14(\mathrm{~m}, 2 \mathrm{H}), 3.94(\mathrm{~s}$, 2H), $2.30(\mathrm{~s}, 3 \mathrm{H}), 1.50(\mathrm{t}, J=7.5 \mathrm{~Hz}, 3 \mathrm{H}) ;{ }^{13} \mathrm{C} \mathrm{NMR}$ $\left(\mathrm{CDCl}_{3}, 150 \mathrm{MHz}\right) \delta$ : For enol-2i: 186.2, 184.4, 136.6, 132.0, 126.1, 122.9, 122.1, 122.0, 113.2, 110.0, 96.6, 41.6, 24.1, 15.0; For keto-2i: 203.4, 187.1, 136.6, 135.4, 126.4, 123.6, 122.9, 122.5, 116.2, 109.8, 57.2, 41.8, 30.1, 15.0; IR (KBr) $v$ : 3444, 3106, 2948, 2924, 2855, 1736, 1642, 1531, $1468,1388,1230 \mathrm{~cm}^{-1}$; HRMS cacld for $\mathrm{C}_{14} \mathrm{H}_{16} \mathrm{NO}_{2}[\mathrm{M}+$ $\mathrm{H}^{+}$230.1176, found 230.1170 .

1-[3-(1-苯甲基吲哚基)]-1,3-丁二酮(2j): 白色固体. m.p. $84 \sim 85{ }^{\circ} \mathrm{C} ;{ }^{1} \mathrm{H}$ NMR $\left(\mathrm{CDCl}_{3}, 600 \mathrm{MHz}\right) \delta$ : For enol-2j: 8.28 (d, $J=7.8 \mathrm{~Hz}, 1 \mathrm{H}), 7.74(\mathrm{~s}, 1 \mathrm{H}), 7.33 \sim 7.23$ (m, 7H), $7.13(\mathrm{~s}, 2 \mathrm{H}), 5.97(\mathrm{~s}, 1 \mathrm{H}), 5.30(\mathrm{~s}, 2 \mathrm{H}), 2.10(\mathrm{~s}$, $3 \mathrm{H})$; For keto-2j: 8.39 (d, $J=7.8 \mathrm{~Hz}, 1 \mathrm{H}), 7.80(\mathrm{~s}, 1 \mathrm{H})$, $7.33 \sim 7.23(\mathrm{~m}, 8 \mathrm{H}), 7.12(\mathrm{~s}, 2 \mathrm{H}), 5.31(\mathrm{~s}, 2 \mathrm{H}), 3.93(\mathrm{~s}$, 2H), $2.30(\mathrm{~s}, 3 \mathrm{H}) ;{ }^{13} \mathrm{C}$ NMR $\left(\mathrm{CDCl}_{3}, 150 \mathrm{MHz}\right) \delta$ : For enol-2j $\delta: 186.4,184.4,137.2,136.3,135.7,129.0$ (2C), 128.2, 127.0 (2C), 126.2, 123.3, 122.3, 122.1, 113.9, 110.4,
96.8, 50.7, 24.1; For keto-2j: 203.2, 187.3, 137.1, 135.4, 132.9, 129.0 (2C), 128.3, 126.9 (2C), 126.4, 123.9, 123.1, 122.6, 115.7, 110.3, 57.1, 50.9, 30.2; IR (KBr) v: 3414, 3113, 3057, 2967, 2930, 2856, 1716, 1634, 1527, 1468, 1387, $1246 \mathrm{~cm}^{-1}$; HRMS cacld for $\mathrm{C}_{19} \mathrm{H}_{18} \mathrm{NO}_{2}[\mathrm{M}+\mathrm{H}]^{+}$ 292.1332, found 292.1334 .

1-[3-(2-甲基- $1 H$-吲哚基)]-3-苯基-1,3-丙二酮(2k): 黄色固体. m.p. $188 \sim 189{ }^{\circ} \mathrm{C} ;{ }^{1} \mathrm{H}$ NMR $\left(\mathrm{CDCl}_{3}, 600\right.$ MHz) $\delta$ : For enol-2k: $8.52(\mathrm{br}, 1 \mathrm{H}), 8.05(\mathrm{~d}, J=7.9 \mathrm{~Hz}$, $1 \mathrm{H}), 7.97$ (d, $J=7.5 \mathrm{~Hz}, 2 \mathrm{H}), 7.52(\mathrm{t}, J=7.4 \mathrm{~Hz}, 1 \mathrm{H}), 7.48$ (t, $J=7.4 \mathrm{~Hz}, 2 \mathrm{H}), 7.32$ (d, $J=7.8 \mathrm{~Hz}, 1 \mathrm{H}), 7.25$ (t, $J=8.8$ $\mathrm{Hz}, 1 \mathrm{H}), 7.22$ (t, $J=7.7 \mathrm{~Hz}, 1 \mathrm{H}), 6.75(\mathrm{~s}, 1 \mathrm{H}), 2.77$ (s, $3 \mathrm{H}) ;{ }^{13} \mathrm{C} \mathrm{NMR}\left(\mathrm{CDCl}_{3}, 150 \mathrm{MHz}\right) \delta$ : For enol-2k: 185.4, 183.1, 142.1, 136.1, 135.0, 131.7, 128.6 (2 C), 126.8 (2C), 126.4, 122.6, 121.9, 120.5, 111.2, 111.0, 95.7, 15.2; IR (KBr) $v: 3419,3225,3120,2980,2830,1773,1734,1598$, 1540, 1456, 1361, 1275, $1242 \mathrm{~cm}^{-1}$; HRMS cacld for $\mathrm{C}_{18} \mathrm{H}_{16} \mathrm{NO}_{2}[\mathrm{M}+\mathrm{H}]^{+}$278.1176; found 278.1280.

1-[3-(1-甲基- $1 H$-吲哚基)]-3-苯基-1,3-丙二酮 (21): 黄色固体. m.p. 106 107 ${ }^{\circ} \mathrm{C} ;{ }^{1} \mathrm{H}$ NMR $\left(\mathrm{CDCl}_{3}, 600\right.$ MHz) $\delta$ : For enol-2I: $8.36 \sim 8.33(\mathrm{~m}, 1 \mathrm{H}), 7.95(\mathrm{~d}, J=7.2$ $\mathrm{Hz}, 2 \mathrm{H}), 7.82(\mathrm{~s}, 1 \mathrm{H}), 7.50(\mathrm{t}, J=7.0 \mathrm{~Hz}, 1 \mathrm{H}), 7.46$ (t, $J=$ $7.0 \mathrm{~Hz}, 3 \mathrm{H}), 7.36 \sim 7.33(\mathrm{~m}, 3 \mathrm{H}), 6.65(\mathrm{~s}, 1 \mathrm{H}), 3.85(\mathrm{~s}$, $3 \mathrm{H}) ;{ }^{13} \mathrm{C} \mathrm{NMR}\left(\mathrm{CDCl}_{3}, 150 \mathrm{MHz}\right) \delta$ : For enol-2I: 185.8 , 179.9, 137.8, 135.7, 134.0, 131.6, 128.6 (2 C), 126.7 (2 C), $126.1,123.3,122.5,122.2,114.2,110.1,93.7,33.7$; IR (KBr) $v$ : 3162, 3115, 2923, 2856, 1717, 1683, 1647, 1541, $1521,1456,1420,1339,1262,1239 \mathrm{~cm}^{-1}$; HRMS cacld for $\mathrm{C}_{18} \mathrm{H}_{16} \mathrm{NO}_{2}[\mathrm{M}+\mathrm{H}]^{+}$278.1176, found 278.1176.

1-(4-甲氧基苯基)-3-[3-(1-甲基- $1 H$-吲哚基)]-1,3-丙 二酮 (2m): 黄色固体. m.p. $145 \sim 146{ }^{\circ} \mathrm{C} ;{ }^{1} \mathrm{H}$ NMR $\left(\mathrm{CDCl}_{3}, 600 \mathrm{MHz}\right) \delta$ : For enol-2m: $8.33(\mathrm{~d}, J=8.5 \mathrm{~Hz}$, $1 \mathrm{H}), 7.90(\mathrm{~d}, J=8.6 \mathrm{~Hz}, 2 \mathrm{H}), 7.77(\mathrm{~s}, 1 \mathrm{H}), 7.30 \sim 7.28(\mathrm{~m}$, $3 \mathrm{H}), 6.92(\mathrm{~d}, J=8.6 \mathrm{~Hz}, 2 \mathrm{H}), 6.55(\mathrm{~s}, 1 \mathrm{H}), 3.83(\mathrm{~s}, 3 \mathrm{H})$, 3.76 (s, 3H); For keto-2m: 8.34 (d, $J=7.5 \mathrm{~Hz}, 1 \mathrm{H}), 8.08$ (d, $J=8.8 \mathrm{~Hz}, 2 \mathrm{H}), 7.91(\mathrm{~s}, 1 \mathrm{H}), 7.26 \sim 7.24(\mathrm{~m}, 3 \mathrm{H}), 6.88$ (d, $J=8.8 \mathrm{~Hz}, 2 \mathrm{H}), 4.38$ (s, 2H), 3.80 (s, 3H), 3.77 (s, 3H); ${ }^{13} \mathrm{C} \mathrm{NMR}\left(\mathrm{CDCl}_{3}, 150 \mathrm{MHz}\right) \delta$ : For enol-2m: 184.5, 180.5, $162.4,137.6,133.6,128.5$ (2C), 128.0, 125.9, 123.0, 122.1, 122.0, 113.8 (2C), 109.9, 109.6, 92.5, 55.9, 33.4; For keto-2m: 193.2, 187.3, 163.8, 137.6, 133.6, 131.7 (2C), 129.5, 126.4 123.5, 122.8, 122.1, 116.0, 113.7 (2C), 109.6, 55.4, 53.0, 33.5. IR (KBr) v: 3432, 3054, 3024, 2972, 2935, 2840, 1792, 1683, 1653, 1599, 1558, 1465, $1368,1259 \mathrm{~cm}^{-1}$; HRMS cacld for $\mathrm{C}_{19} \mathrm{H}_{18} \mathrm{NO}_{3}[\mathrm{M}+\mathrm{H}]^{+}$ 
308.1281, found 308.1286.

1-(4-溴苯基)-3-[3-(1-甲基- $1 H$-吲哚基)]-1,3-丙二酮 (2n): 黄色固体. m.p. $165 \sim 166{ }^{\circ} \mathrm{C} ;{ }^{1} \mathrm{H}$ NMR $\left(\mathrm{CDCl}_{3}\right.$, $600 \mathrm{MHz}) \delta$ : For enol-2n: $8.30(\mathrm{~d}, J=8.2 \mathrm{~Hz}, 1 \mathrm{H}), 7.74$ (d, $J=8.4 \mathrm{~Hz}, 2 \mathrm{H}), 7.72(\mathrm{~s}, 1 \mathrm{H}), 7.54(\mathrm{~d}, J=8.3 \mathrm{~Hz}, 2 \mathrm{H})$, $7.31 \sim 7.27(\mathrm{~m}, 3 \mathrm{H}), 6.52(\mathrm{~s}, 1 \mathrm{H}), 3.78(\mathrm{~s}, 3 \mathrm{H})$; For keto-2n: 8.31 (d, $J=8.2 \mathrm{~Hz}, 1 \mathrm{H}), 8.08$ (d, $J=8.4 \mathrm{~Hz}, 2 \mathrm{H})$, $7.88(\mathrm{~s}, 1 \mathrm{H}), 7.56(\mathrm{~d}, J=8.6 \mathrm{~Hz}, 2 \mathrm{H}), 7.31 \sim 7.27(\mathrm{~m}, 3 \mathrm{H})$, $4.40(\mathrm{~s}, 2 \mathrm{H}), 3.79(\mathrm{~s}, 3 \mathrm{H}) ;{ }^{13} \mathrm{C} \mathrm{NMR}\left(\mathrm{CDCl}_{3}, 150 \mathrm{MHz}\right) \delta$ : For enol-2n: 185.9, 178.4, 137.7, 134.5, 134.2, 131.8 (2C), 128.1 (2C), 126.1, 126.0, 123.4, 122.5, 122.2, 114.0, 110.1, 93.4, 33.6; For keto-2n: 193.9, 186.7, 137.5, 135.1, 134.2, 132.0 (2C), 130.9 (2C), 129.0, 126.4, 123.8, 123.1, 122.5, 116.0, 109.8, 53.3, 33.7; IR (KBr) v: 3400, 3107, 3069, 2948, 2911, 2880, 1782, 1607, 1560, 1523, 1465, 1359, $1222 \mathrm{~cm}^{-1}$; HRMS cacld for $\mathrm{C}_{18} \mathrm{H}_{15} \mathrm{BrNO}_{2}[\mathrm{M}+\mathrm{H}]^{+}$ 356.0281, found 356.0289.

1-[3-(1-甲基-1H-吲哚基)]-3-(2-菜基)-1,3-丙二酮 (2o): 黄色粘稠液体. ${ }^{1} \mathrm{H} \mathrm{NMR}\left(\mathrm{CDCl}_{3}, 600 \mathrm{MHz}\right) \delta$ : For enol-2o: 8.53 (d, $J=8.4 \mathrm{~Hz}, 1 \mathrm{H}), 7.74(\mathrm{~d}, J=8.4 \mathrm{~Hz}, 2 \mathrm{H})$, $8.31(\mathrm{t}, J=8.2 \mathrm{~Hz}, 1 \mathrm{H}), 7.92$ (d, $J=8.1 \mathrm{~Hz}, 1 \mathrm{H}), 7.88$ (d, $J=8.0 \mathrm{~Hz}, 1 \mathrm{H}), 7.73(\mathrm{~s}, 1 \mathrm{H}), 7.56(\mathrm{t}, J=7.1 \mathrm{~Hz}, 1 \mathrm{H})$, $7.53 \sim 7.49(\mathrm{~m}, 2 \mathrm{H}), 7.33 \sim 7.30(\mathrm{~m}, 3 \mathrm{H}), 6.45(\mathrm{~s}, 1 \mathrm{H})$, $3.79(\mathrm{~s}, 3 \mathrm{H}) ;{ }^{13} \mathrm{C} \mathrm{NMR}\left(\mathrm{CDCl}_{3}, 150 \mathrm{MHz}\right) \delta$ : For enol-2o: $185.1,183.8,137.7,134.9,134.1,133.8,130.8,130.3$, $128.4,126.9,126.5,126.2,125.7,124.9,123.3122 .9$, $122.4,122.1,113.7,110.0,98.2$, 33.5; IR (KBr) v: 3393, 3117, 3052, 2926, 2847, 1724, 1644, 1540, 1466, 1364, $1221 \mathrm{~cm}^{-1}$; HRMS cacld for $\mathrm{C}_{22} \mathrm{H}_{18} \mathrm{NO}_{2}[\mathrm{M}+\mathrm{H}]^{+}$ 328.1332, found 328.1337 .

1-[3-(1-甲基- $1 H$-吲哚基)]-3-(2-噻吩基)-1,3-丙二酮 (2p): 黄色固体. m.p. $108 \sim 109{ }^{\circ} \mathrm{C}$; ${ }^{1} \mathrm{H}$ NMR $\left(\mathrm{CDCl}_{3}\right.$, $600 \mathrm{MHz}) \delta$ : For enol-2p: $8.23(\mathrm{t}, J=8.2 \mathrm{~Hz}, 1 \mathrm{H}), 7.75(\mathrm{~s}$, $1 \mathrm{H}), 7.72$ (d, $J=3.2 \mathrm{~Hz}, 1 \mathrm{H}), 7.53$ (d, $J=4.7 \mathrm{~Hz}, 1 \mathrm{H})$, $7.32 \sim 7.30(\mathrm{~m}, 2 \mathrm{H}), 7.29(\mathrm{~s}, 1 \mathrm{H}), 7.13 \sim 7.10(\mathrm{~m}, 1 \mathrm{H}), 6.5$ (s, 1H), $3.81(\mathrm{~s}, 3 \mathrm{H})$; For keto-2p: $8.34(\mathrm{t}, J=8.1 \mathrm{~Hz}, 1 \mathrm{H})$, $8.01(\mathrm{~d}, J=3.4 \mathrm{~Hz}, 1 \mathrm{H}), 7.96(\mathrm{~s}, 1 \mathrm{H}), 7.63$ (d, $J=3.2 \mathrm{~Hz}$, $1 \mathrm{H}), 7.32 \sim 7.30(\mathrm{~m}, 2 \mathrm{H}), 7.29(\mathrm{~s}, 1 \mathrm{H}), 7.13 \sim 7.10(\mathrm{~m}$, $1 \mathrm{H}), 4.37(\mathrm{~s}, 2 \mathrm{H}), 3.80(\mathrm{~s}, 3 \mathrm{H}) ;{ }^{13} \mathrm{C} \mathrm{NMR}\left(\mathrm{CDCl}_{3}, 150\right.$ $\mathrm{MHz}) \delta$ : For enol-2p: 181.5, 178.2, 141.9, 137.8, 134.9 (2C), 130.7, 128.8, 125.7, 123.1, 122.2, 121.8, 112.5, 110.1, 92.7, 33.5; For keto-2p: 187.2, 186.3, 143.7, 137.7, $137.4,133.6,128.5,128.1,126.4,123.7,122.9,122.5$, 115.9, 109.7, 54.4, 33.6; IR (KBr) v: 3420, 3111, 3053, 2936, 2889, 1792, 1627, 1533, 1469, 1369, $1230 \mathrm{~cm}^{-1}$;
HRMS cacld for $\mathrm{C}_{16} \mathrm{H}_{14} \mathrm{NO}_{2} \mathrm{~S}[\mathrm{M}+\mathrm{H}]^{+}$284.0740, found 284.0745 .

Supporting Information 化合物 2 的 ${ }^{1} \mathrm{H}$ NMR 和 ${ }^{13} \mathrm{C}$ NMR．这些材料可以免费从本刊网站(http://sioc-journal.cn/)上下载.

\section{References}

[1] Goldgur, Y.; Craigie, R.; Cohen, G. H.; Fujiwara, T.; Yoshinaga, T.; Fujishita, T.; Sugimoto, H.; Endo, T.; Murai, H.; Davies, D. R. Proc. Natl. Acad. Sci. U. S. A. 1999, 96, 13040.

[2] Herr, R. J. Bioorg. Med. Chem. 2002, 10, 3379.

[3] Jain, S. V.; Sonawane, L. V.; Patil, R. R.; Bari, S. B. Med. Chem. Res. 2012, 21, 165

[4] Zhang, D. T.; Wang, G. T.; Tan, C. B.; Xu, W. R.; Pei, Y.; Huo, L. Y. Arch. Pharm. Res. 2011, 34, 343.

[5] Diana, P.; Carbone, A.; Barraja, P.; Kelter, G.; Fiebig, H. H.; Cirrincione, G. Bioorg. Med. Chem. 2010, 18, 4524

[6] Carbone, A.; Spanò, V.; Parrino, B.; Ciancimino, C.; Attanasi, O. A.; Favi, G. Molecules 2013, 18, 2518.

[7] Bergman, J. Acta Chem. Scand. 1968, 22, 1063.

[8] Pais, G. C. G.; Zhang, X. C.; Marchand, C.; Neamati, N.; Cowansage, K.; Svarovskaia E. S.; Pathak, V. K.; Tang, Y.; Nicklaus, M.; Pommier, Y.; Burke, T. R. J. Med. Chem. 2002, 45, 3184.

[9] Kostryukova, T. S.; Ivanovskaya, N. P.; Lyamin, A. I.; Romanov, D. V.; Osin, N. S.; Zatonsky, G. V.; Vasil'ev N. V. Russ. J. Gen. Chem. 2012, 82, 455 .

[10] Yu, H. F.; Li, T. C.; Liao, P. Q.; Diao, Q. P.; Xin, G.; Hou, D. Y. Chin. J. Org. Chem. 2014, 34, 956 (in Chinese) (于海丰, 李铁纯, 廖沛球, 刀全平, 辛广, 侯冬岩, 有机化学, 2014, 34, 956.)

[11] Yu, H. F.; Liao, P. Q.; Diao, Q. P.; Li, T. C.; Xin, G.; Han, L. N.; Hou, D. Y. Chin. J. Org. Chem. 2014, 34, 1851 (in Chinese) (于海丰, 廖沛球, 刀全平, 李铁纯，辛广，韩立楠，侯冬岩，有 机化学, 2014, 34, 1851.)

[12] Wang, W. J.; Yu, H. F. Synth. Commun. 2019, 49, 377.

[13] Kobayashi, S.; Manabe, K. Acc. Chem. Res. 2002, 35, 209.

[14] Zhou, Z.; Duan, J. F.; Mu, X. J.; Xiao, S. Y. Chin. J. Org. Chem. 2018, 38, 585 (in Chinese). (周基，段建凤，穆小静，肖尚有，有机化学, 2018, 38, 585.)

[15] Xu, Z. H.; Chen, F. B.; Li, Y. Y.; Huang, Q. S.; Liao, C. W. Chin. J. Org. Chem. 2018, 38, 3101 (in Chinese).

(许招会, 陈飞彪, 李瑜钰, 黄清水, 廖传文, 有机化学, 2018, 38, 3101.)

[16] Wang, Z.; Yang, L.; Liu, H. L.; Bao, W. H.; Tan, Y. H.; Wang, M.; Tang, Z. L.; He, W. M. Chin. J. Org. Chem. 2018, 38, 2639 (in Chinese).

(王峥，杨柳，刘慧兰，谭英芝，包文虎，汪明，唐子龙，何卫民， 有机化学, 2018, 38, 2639.)

[17] Xie, L. Y.; Li, Y. J.; Qu, J.; Duan, Y.; Hu, J.; Liu, K. J.; Cao, Z.; He, W. M. Green Chem. 2017, 19, 5642.

[18] Xie, L. Y.; Peng, S.; Tan, J. X.; Sun, R. X.; Yu, X. Y.; Dai, N. N.; Tang, Z. L.; Xu, X. H.; He, W. M. ACS Sustainable Chem. Eng. 2018, 6, 16976.

[19] Xie, L. Y.; Peng, S.; Liu, F.; Liu, Y. F.; Sun, M.; Tang, Z. L.; Jiang, S.; Cao, Z.; He, W. M. ACS Sustainable Chem. Eng. 2019, 77, 7193.

[20] Dong, D. W.; Ouyang, Y.; Yu, H. F.; Liu, Q.; Liu, J.; Wang, M.; Zhu, J. J. Org. Chem. 2005, 70, 4535. 
[21] Yu, H. F.; Liao, P. Q. Tetrahedron Lett. 2016, 57, 2868

[22] Qi, F.; Yu, H. F.; Wang, Y. N.; Lv, Y.; Li, Y. X.; Han, L.; Wang, R.; Feng, X. N. Synth. Commun. 2017, 47, 2220.

[23] Pan, L.; Bi, X. H.; Liu, Q. Chem. Soc. Rev. 2013, 42, 1251.

[24] Yu, H. F.; Zhao, L. J.; Diao, Q. P.; Li, T. C.; Liao, P. Q.; Hou, D. Y.; Xin, G. Synlett 2017, 28, 1828.

[25] Zhao H.; Diao, Q. P.; Yu, H. F.; Li, T. C.; Liao, P. Q.; Hou, D. Y. Chem. Res. Chin. Univ. 2017, 33, 746.
[26] Yu, H. F.; Jin, W. W.; Sun, C. L.; Yu, Z. K. Angew. Chem., Int. Ed. 2010, 49, 5792.

[27] Yu, H. F.; Yu, Z. K. Angew. Chem., Int. Ed. 2009, 48, 2929.

[28] Yu, H. F.; Li, T. C.; Liao, P. Q. Synthesis 2012, 44, 3743.

[29] Wang, Q.; Sun, Y. X.; Cui, S. Y.; Zhao, H.; Huang, W.; Xie, X. H. J. Mianyang Teachers' College 2017, 36, 30 (in Chinese).

(王群, 孙玉希, 崔书亚, 赵洪, 黄文, 谢欣宏, 绵阳师范学院学 报, 2017, 36, 30.) 